\title{
The Microbiome and Allogeneic Stem Cell Transplantation
}

\author{
Daniela Sporrer • Andre Gessner • Thomas Hehlgans • \\ Peter J. Oefner • Ernst Holler
}

Published online: 15 January 2015

(C) Springer International Publishing AG 2015

\begin{abstract}
Graft-versus-host disease (GvHD) is the major cause of mortality and morbidity after allogeneic stem cell transplantation. Recently, deep sequencing of the hypervariable regions of the 16S rRNA gene in stool specimens significantly enhanced our understanding of a likely pathophysiologic role of the microbiome in GvHD. Here, we summarize the indirect and direct evidence for a microbiome involvement. Indirect evidence for a role of the intestinal microbiota has been provided by experiments in germ-free mice and single nucleotide polymorphism analysis of receptors of innate
\end{abstract}

This article is part of the Topical Collection on Microbiome and Stem Cell Function

D. Sporrer $\cdot$ E. Holler

Department of Internal Medicine 3, University Medical Center,

93042 Regensburg, Germany

D. Sporrer

e-mail: Daniela.sporrer@ukr.de

\author{
A. Gessner \\ Institute of Clinical Microbiology and Hygiene, University Medical \\ Center, 93042 Regensburg, Germany \\ e-mail: Andre.gessner@ukr.de \\ T. Hehlgans \\ Department of Immunology, University Medical Center, \\ 93042 Regensburg, Germany \\ e-mail: Thomas.Hehlgans@ukr.de \\ P. J. Oefner \\ Institute of Functional Genomics, University of Regensburg, \\ 93053 Regensburg, Germany \\ e-mail: Peter.oefner@ukr.de \\ E. Holler $(\bowtie)$ \\ Regensburg Center for Interventional Immunology, \\ 93042 Regensburg, Germany \\ e-mail: Ernst.holler@ukr.de
}

immunity. Direct 16S rRNA sequencing showed a substantial loss of diversity in stool specimens from patients with GvHD, which is associated with inferior outcomes. We discuss mechanisms such as interference with antimicrobial peptides and potential approaches to reduce complications by microbiome modulation.

Keywords Allogeneic stem cell transplantation · GvHD . Microbiome $\cdot$ Receptors of innate immunity $\cdot$ Intestinal immune regulation $\cdot$ Antimicrobial peptides

\section{Introduction}

Graft-versus-host disease (GvHD) is the major cause of transplant-related mortality and morbidity following allogeneic stem cell transplantation (SCT), which has become an otherwise curative treatment for a variety of nonmalignant and malignant hematologic diseases. GvHD results from the activation of donor $\mathrm{T}$ lymphocytes, which are transplanted together with hematopoietic precursors and stem cells; activation is initiated by histocompatibility antigens presented on host antigen-presenting cells (APCs) and target tissues. Activated donor $\mathrm{T}$ cells proliferate, expand, and induce apoptotic damage in epithelial targets either directly or by induction of effector cells and cytokines [1]. However, for full antigen presentation and donor cell activation, inflammatory checkpoints must be triggered, as elegantly shown by Chakraverty et al. [2] in a murine GvHD model, in which prior Toll-like receptor (TLR) stimulation was needed to induce GvHD in target organs. The broadest set of inflammatory triggers may be provided by the human microbiome; therefore, we summarize here our current knowledge and concepts regarding its role in GvHD. 


\section{Historical Aspects: GvHD and the Intestinal Microflora}

The observation that acute GvHD primarily targets the skin, gastrointestinal tract, and liver - with chronic GvHD affecting the oral cavity, urogenital tract, eyes, lungs, and other organs - offered the first clue to a potential role of the microbial environment in the pathogenesis of GvHD. Almost all these target organs have epithelial interfaces between the microbial environment and the host immune system, which might explain the tropism of GvHD to a large extent.

The first clear demonstration of a major role of the intestinal microbiome or microbiota in GvHD, at least in gastrointestinal GvHD, came from the seminal observation by van Bekkum et al. and van Bekkum and Knaan [3, 4] that mice grown and kept under germ-free conditions up to 40 days after transplantation did not develop GvHD, whereas early microbial recolonization of the gut resulted in a marked increase in mortality. However, the authors also showed an interplay between the dose of $\mathrm{T}$ cells and microbial activation, as mice receiving bone marrow and splenocytes as an additional $\mathrm{T}$ cell source were not fully protected by a germ-free environment but showed delayed GvHD. van Bekkum's observation provided a major argument for introducing gnotobiotic approaches to clinical transplantation in the early 1980s [5, 6], and attempts toward either total or selective decontamination, along with protective isolation of patients, have been the standard of clinical care for decades in most clinical transplant centers $[7,8]$.

\section{Indirect Evidence for a Role of the Microbiome in GvHD}

Over the years, several hypotheses have been put forward regarding the role of the microbiota in the pathogenesis of GvHD. First, cross-reactions of bacterial and histocompatibility antigens were postulated. After the discovery of lipopolysaccharide (LPS), an LPS-APC axis resulting in excess activation of APCs was the favorite explanation [9, 10]. Description of TLRs and NOD-like receptors (NLRs) soon suggested a far more complex interaction between different microbial pathogen-associated molecular patterns (PAMPs) and the immune system. Studies of TLR and NOD knockout mice used as recipients and donors in SCT models [11, 12], as well as human single nucleotide polymorphisms (SNPs) resulting in functional defects of pathogen-receptor interaction, have demonstrated an association of TLRs and NLRs with GvHD [13-15]. Clinical studies with SNPs of NOD2/CARD15 showed wide heterogeneity among individual center-specific cohorts [16-25], and differences in the strategies used for intestinal decontamination, which seemed to be a major discriminating factor among these centers [26]. This again suggests a major role of the intestinal microbiome. Strategies to neutralize endotoxin via polyclonal and monoclonal antibodies attenuated GvHD pathology in experimental models $[27,28]$ and vice versa, and experimental modulation of the gut bacteria via Lactobacillus rhamnosus reduced the severity of GvHD pathology, prevented bacterial translocation into mesenteric lymph nodes, and resulted in increased survival [29].

\section{Direct Evidence for a Role of the Microbiome in GvHD}

In the past 10 years, metagenomic and other cultureindependent analyses of the microbiome became available based on next-generation sequencing of the $16 \mathrm{~S}$ ribosomal RNA genes. This technique not only prompted a large series of studies investigating the general role of the microbiome in health and disease [30 $0^{\circ}$ but also allowed a more specific examination of the role of the gastrointestinal microbiome in allogeneic SCT. In a pivotal study, the group of van den Brink $[31 \bullet \bullet]$ reported the loss of microbiome diversity in mice developing GvHD. Furthermore, antibiotic pretreatment before conditioning induced a microbiome shift that was aggravated in GvHD and associated with an increased pathology score as well as reduced survival. Similarly, stool specimens collected from patients revealed a loss of diversity, as indicated by the Shannon index after transplantation, which was more pronounced in patients with GvHD. A major loss occurred within Clostridiales, whereas Lactobacillales significantly increased in GvHD patients. In a pilot study assessing microbiome changes in 32 patients undergoing allogeneic SCT at our unit [32॰], we also observed the near disappearance of commensal bacteria such as clostridia and a shift toward enterococci in the first weeks after SCT. These shifts were partially induced by decontamination using quinolones and metronidazole; however, enterococcal abundances also were related to early intestinal GvHD. We used not only $16 \mathrm{~S}$ rRNA sequencing and Enterococcus-specific PCR but also analysis of urine indoxyl sulfate (IS), which is synthesized in the liver from indole produced by the intestinal microbiota that process tryptophan in dietary proteins. Again, we observed suppression of urinary IS levels by decontamination and antibiotics, which was most pronounced in patients developing intestinal GvHD. Finally, Taur et al. [33••] extended their observations to 82 patients and reported a strong association between microbiome diversity soon after engraftment and 3-year nonrelapse mortality, which also was significant in a multivariate analysis, suggesting a strong pathophysiologic involvement of loss of diversity of the microbiome. In their stool specimens, patients with high diversity had heterogeneous patterns of species, including a predominance of Blautia, whereas similar to our study, enterococci-among other pathogenic species - frequently dominated in patients with low diversity. We recently reanalyzed the original microbiome results of our pilot study, addressing the question of whether 
specific species were associated with improved survival. Interestingly, patients exhibiting a higher proportion of Clostridiales, especially Eubacterium rectale, in the first 4 weeks after transplantation had significantly lower nonrelapse-related mortality than patients with suppression (Fig. 1), thus supporting the data reported by Taur et al. [33••]. Of course, our data need confirmation in a larger, prospective study allowing multivariate analysis.

\section{Pathophysiologic Considerations}

Although the association of loss of diversity in the intestinal microbiome with GvHD and increased mortality is now confirmed by independent studies in both mice and humans, the exact pathophysiology and sequence of events behind this observation need clarification. The early occurrence of loss of diversity makes it difficult to determine whether loss of diversity precedes GvHD or rather is a consequence of it. Current concepts, as summarized in Table 1, support both theories.

Major determinants of microbiome composition are antimicrobial peptides (AMPs), which are produced by specialized cells, such as the Paneth cells, in the small intestine but also by neutrophils and, in the case of $\beta$-defensins, by epithelial cells in general [34]. AMP induction is regulated by

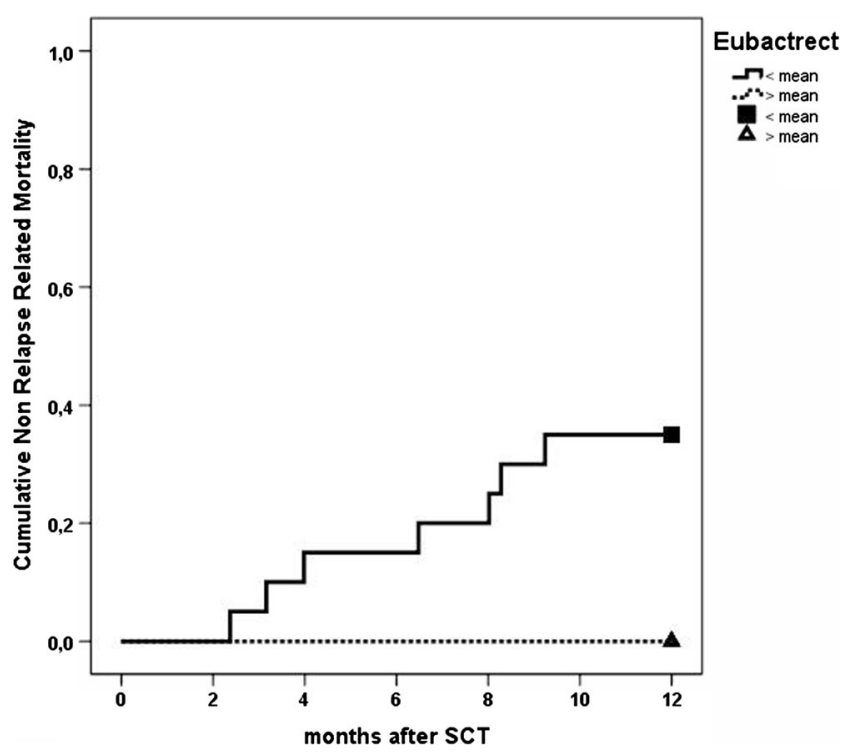

Fig. 1 Stool specimens from days 0 to 21 were subjected to deep sequencing of the hypervariable region V3 of the 16S rRNA gene, and the mean fraction of $E$. rectale was calculated for each patient. Patients were grouped according to mean $E$. rectale content below $(n=20)$ or above $(n=10)$ the mean of all patients, and nonrelapse related mortality (NRM) was calculated for both groups. The outcome was of borderline significance based on log-rank analysis $(P=0.04)$
Table 1 Potential pathophysiologic mechanisms explaining the association between loss of diversity and poor outcome following allogeneic SCT

\begin{tabular}{lll}
\hline Sequence & Etiology & Mechanism of action \\
\hline $\begin{array}{c}\text { Loss of diversity } \rightarrow \\
\text { GvHD }\end{array}$ & $\begin{array}{c}\text { Broad-spectrum } \\
\text { antibiotics } \\
\text { NOD2 deficiency }\end{array}$ & $\begin{array}{c}\text { Suppression of commensal } \\
\text { bacteria required for AMPs } \\
\text { Diminished and altered } \\
\text { production of AMPs } \\
\text { Direct damage to epithelial } \\
\text { stem cells, including } \\
\text { Paneth cells }\end{array}$ \\
& Loss of diversity & $\begin{array}{c}\text { Impaired ILC function and } \\
\text { impaired production of } \\
\text { protective IL-22 }\end{array}$ \\
& Direct Paneth cell & $\begin{array}{c}\text { Diminished local protection } \\
\text { by AMPs }\end{array}$ \\
diversity & Loss of diversity & Impaired Treg function (directly \\
& & $\begin{array}{l}\text { and via suppression of } \\
\text { butyrate production by } \\
\text { commensal bacteria) }\end{array}$ \\
& &
\end{tabular}

commensal bacteria. Further, the broad-spectrum antibiotics administered to most patients during the early neutropenic period contribute to loss of diversity directly and indirectly via alteration of AMPs. Similar effects occur in relation to antibiotic decontamination, as shown in our study. Because NOD2 SNPs also affect microbiome composition, again via diminished activity of Paneth cell peptides, changes in microbiome diversity may at least partially explain the association between NOD2/CARD15 SNPs and intestinal GvHD $[35,36 \bullet \cdot, 37]$.

There is increasing evidence that a diverse microbiome is a prerequisite for the maintenance of immunologic homeostasis in epithelial tissues. Commensal bacteria are needed to induce type 3 innate lymphoid cells (ILCs), which maintain epithelial integrity via interleukin $22[38,39]$. Recent studies strongly suggest involvement of ILCs in GvHD [40•, 41]. Furthermore, the high proportion of regulatory $\mathrm{T}$ (Treg) cells in epithelial tissues may be linked directly to the presence of specific bacteria. Actual studies show that short-chain fatty acids such as butyrate, which are produced by commensal colonic bacteria, support not only expansion of nonthymic Treg cells $[42,43,44 \cdot \bullet]$ but also direct recognition of bacterial antigens by Treg cells, suggesting that these cells are actively involved in the immunologic tolerance of the healthy microbiome $[45,46]$. Interference with both ILCs and Treg cells, by loss of diversity, should facilitate activation of alloreactive $\mathrm{T}$ cells in GvHD, at least by amplifying inflammation.

Paneth cells have been addressed only recently in GvHD pathology. Eriguchi et al. [47••] were the first to describe the role of these cells in murine models of SCT, and they 
demonstrated the direct destruction of Paneth cells by GvHD with subsequent loss of AMPs and microbiome changes. In clinical GvHD, Paneth cells were identified as potential targets by the description of Reg3alpha, a Paneth cell peptide, as a highly specific marker of intestinal GvHD [48]. In a cooperative pathology study with colleagues from Ann Arbor, we found direct evidence of GvHD-dependent Paneth cell damage in upper gastrointestinal GvHD, which was highly predictive of poor outcome [49•].

Thus, there is a mutual influence of loss of diversity on intestinal immune regulation and of GvHD on loss of diversity, which may be considered a vicious circle amplifying GvHD-related tissue damage, which may explain the strong impact of loss of diversity on outcome.

\section{Further Impact of Microbiome Changes: Infections}

Besides involvement in inflammation and alloreaction, another important aspect is the concept that the microbiome sets the stage for infections in these heavily immunosuppressed patients. The New York group addressed this question and reported that greater than $30 \%$ domination of the intestinal microbiome by enterococci increased the risk of bacteremia with vancomycin-resistant enterococci ninefold, whereas domination of the microbiome by Proteobacteria was associated with a fivefold risk of bacteremia with Gram-negative rods [50]. These data further support the concept that translocation of intestinal bacteria is a major mechanism of bacterial infection in SCT patients. In a small pilot study addressing the oral microbiome in 11 SCT patients, Ames et al. [51] did not only observe major shifts of the oral microbiome after SCT but also reported changes in the microbiome in association with respiratory infections.

\section{Future Directions}

\section{The Fate of Decontamination}

An interesting but unanswered question is whether the aforementioned observations argue against the concept of complete decontamination to reduce GvHD and thus improve outcome [52]. Whereas loss of diversity might indicate inappropriate decontamination and loss of protective bacteria, the group of Leiden recently reconfirmed their observation on complete decontamination in children undergoing SCT [53]. Complete decontamination, as documented by cultural approaches, also was a predictive and independent factor preventing GvHD in a series of children receiving allogeneic SCT between 1989 and 2003. However, molecular studies addressing the exact extent of decontamination on the basis of 16S rRNA sequencing are lacking and urgently needed to answer the question whether complete decontamination can be achieved at all and, if so, whether complete decontamination is more desirable than maintenance of commensal protective bacteria. Considering the increasing clinical relevance of multiresistant bacteria in hospitalized patients and the potential selection of these bacteria by loss of diversity, decontamination and antibiotic prophylaxis must be reconsidered and reevaluated urgently by molecular approaches.

\section{Microbiome Modulation to Prevent or Treat GvHD}

If loss of diversity predicts poor outcome, any attempt to avoid it or to restore diversity should lead to reduced intestinal inflammation and GvHD. Prophylactic use of probiotics is suggested by our experimental data on the use of L. rhamnosus in a murine model [29]. However, probiotics may carry the risk of lethal septicemia in patients with neutropenia. Prebiotic approaches, such as introducing plant fibers to promote butyrate-producing colonic bacteria, may be safer [54]. An interesting alternative explanation for the association of microbiome changes with GvHD is the hypothesis that these changes merely reflect the impaired oral uptake of nutrition, which itself has a major impact on epithelial integrity and inflammation; this hypothesis also needs further evaluation. Protection by oral nutrition may partially explain the reduced GvHD incidence and improved outcome in patients receiving allogeneic transplantation under homecare conditions, as reported by the Huddinge group $[55,56]$. Direct microbiota transplantation would be the ultimate approach to restore microbial diversity. If the immune system interacts directly with the intestinal microbiome, the role of donor versus recipient microbiota has to be evaluated in this context. So far, only one murine study addressed the impact of donor microbiome on GvHD, but it did not observe a major influence [57]. Other options to modulate intestinal inflammation include restoration of epithelial integrity, topical application of AMPs, and promotion of regulatory immune cells, such as ILCs, as reported by Hanash et al. [40•].

\section{Conclusions and Other GvHD Target Organs and Pathogens}

As indicated in the "Introduction," GvHD mainly targets epithelial tissues exposed to direct interaction with the respective microbiome. Among these, the skin, lungs and bronchi, oral mucosa, and urogenital tract are affected most severely by both acute and chronic GvHD, and the impact of the microbiome on the pathophysiology of organ GvHD must be characterized in the coming years. Interestingly, the pathophysiology of liver diseases increasingly is linked to changes 
in the gut microbiome and thus may reflect an impaired interaction with the intestinal microbiome or PAMPs released into the portal vein [58].

Given the strong interaction of the host immune system not only with bacteria but also with viruses and fungal species, molecular techniques will have to be adapted to the characterization of both the microbiome and the virome and their interaction in GvHD.

Acknowledgments This work was partially supported by the German Research Foundation (KFO243 [E.H. and T.H.] and SPP1656 [A.G.]).

\section{Compliance with Ethics Guidelines}

Conflict of Interest Daniela Sporrer, Andre Gessner, Thomas Hehlgans, Peter J. Oefner, and Ernst Holler declare that they have no conflict of interest.

Human and Animal Rights and Informed Consent Figure 1 contains data, which were collected within a clinical diagnostic trial. All procedures including analysis of the stool microbiome in the course of allogeneic transplantation and data collection were in accordance with the ethical standards of the institutional research committee and with the 1964 Declaration of Helsinki and its later amendments. The study was approved by the local ethical review board, and informed consent was obtained from all individual participants included in the study.

\section{References}

Papers of particular interest, published recently, have been highlighted as:

- Of importance

•• Of major importance

1. Ferrara JL, Levine JE, Reddy P, Holler E. Graft-versus-host disease. Lancet. 2009;373(9674):1550-61.

2. Chakraverty R, Côté D, Buchli J, Cotter P, Hsu R, Zhao G, et al. An inflammatory checkpoint regulates recruitment of graft-versus-host reactive T cells to peripheral tissues. J Exp Med. 2006;203(8): 2021-31.

3. van Bekkum DW, Roodenburg J, Heidt PJ, van der Waaij D. Mitigation of secondary disease of allogeneic mouse radiation chimeras by modification of the intestinal microflora. J Natl Cancer Inst. 1974;52(2):401-4.

4. van Bekkum DW, Knaan S. Role of bacterial microflora in development of intestinal lesions from graft-versus-host reaction. J Natl Cancer Inst. 1977;58(3):787-90.

5. Heidt PJ, Vossen JM. Experimental and clinical gnotobiotics: influence of the microflora on graft-versus-host disease after allogeneic bone marrow transplantation. J Med. 1992;23(3-4):161-73.

6. Vossen JM, Heidt PJ, van den Berg H, Gerritsen EJ, Hermans J, Dooren LJ. Prevention of infection and graft-versus-host disease by suppression of intestinal microflora in children treated with allogeneic bone marrow transplantation. Eur J Clin Microbiol Infect Dis. 1990;9(1):14-23.

7. Passweg JR, Rowlings PA, Atkinson KA, Barrett AJ, Gale RP, Gratwohl A, et al. Influence of protective isolation on outcome of allogeneic bone marrow transplantation for leukemia. Bone Marrow Transplant. 1998;21(12):1231-8.
8. Beelen DW, Elmaagacli A, Müller KD, Hirche H, Schaefer UW. Influence of intestinal bacterial decontamination using metronidazole and ciprofloxacin or ciprofloxacin alone on the development of acute graft-versus-host disease after marrow transplantation in patients with hematologic malignancies: final results and long-term follow-up of an open-label prospective randomized trial. Blood. 1999;93(10):3267-75.

9. Cooke KR, Olkiewicz K, Erickson N, Ferrara JL. The role of endotoxin and the innate immune response in the pathophysiology of acute graft versus host disease. J Endotoxin Res. 2002;8(6):441-8.

10. Cooke KR, Gerbitz A, Crawford JM, Teshima T, Hill GR, Tesolin A, et al. LPS antagonism reduces graft-versus-host disease and preserves graft-versus-leukemia activity after experimental bone marrow transplantation. J Clin Invest. 2001;107(12):1581-9.

11. Heimesaat MM, Nogai A, Bereswill S, Plickert R, Fischer A, Loddenkemper C, et al. MyD88/TLR9 mediated immunopathology and gut microbiota dynamics in a novel murine model of intestinal graft-versus-host disease. Gut. 2010;59(8):1079-87.

12. Penack O, Holler E, van den Brink MR. Graft-versus-host disease: regulation by microbe-associated molecules and innate immune receptors. Blood. 2010;115(10):1865-72.

13. Lorenz E, Schwartz DA, Martin PJ, Gooley T, Lin MT, Chien JW, et al. Association of TLR4 mutations and the risk for acute GVHD after HLA-matched-sibling hematopoietic stem cell transplantation. Biol Blood Marrow Transplant. 2001;7(7):384-7.

14. Elmaagacli AH, Koldehoff M, Hindahl H, Steckel NK, Trenschel $\mathrm{R}$, Peceny $\mathrm{R}$, et al. Mutations in innate immune system NOD2/CARD 15 and TLR-4 (Thr399Ile) genes influence the risk for severe acute graft-versus-host disease in patients who underwent an allogeneic transplantation. Transplantation. 2006;81(2):247-54.

15. Xiao HW, Luo Y, Lai XY, Shi JM, Tan YM, He JS, et al. Donor TLR9 gene tagSNPs influence susceptibility to aGVHD and CMV reactivation in the allo-HSCT setting without polymorphisms in the TLR4 and NOD2 genes. Bone Marrow Transplant. 2014;49(2): 241-7.

16. Jaskula E, Lange A, Kyrcz-Krzemien S, Markiewicz M, DzierzakMietla M, Jedrzejczak WW, et al. Polish Donor-Recipient Matching Group. NOD2/CARD15 single nucleotide polymorphism 13 (3020insC) is associated with risk of sepsis and single nucleotide polymorphism $8(2104 \mathrm{C}>\mathrm{T})$ with herpes viruses reactivation in patients after allogeneic hematopoietic stem cell transplantation. Biol Blood Marrow Transplant. 2014;20(3):409-14.

17. Kreyenberg H, Jarisch A, Bayer C, Schuster B, Willasch A, Strahm $\mathrm{B}$, et al. NOD2/CARD15 gene polymorphisms affect outcome in pediatric allogeneic stem cell transplantation. Blood. 2011;118(4): $1181-4$.

18. van der Straaten HM, Paquay MM, Tilanus MG, van Geloven N, Verdonck LF, Huisman C. NOD2/CARD15 variants are not a risk factor for clinical outcome after nonmyeloablative allogeneic stem cell transplantation. Biol Blood Marrow Transplant. 2011;17(8): 1231-6.

19. Wermke M, Maiwald S, Schmelz R, Thiede C, Schetelig J, Ehninger G, et al. Genetic variations of interleukin-23R $(1143 \mathrm{~A}>\mathrm{G})$ and BPI (A645G), but not of NOD2, are associated with acute graft-versus-host disease after allogeneic transplantation. Biol Blood Marrow Transplant. 2010;16(12):1718-27.

20. Gruhn B, Intek J, Pfaffendorf N, Zell R, Corbacioglu S, Zintl F, et al. Polymorphism of interleukin-23 receptor gene but not of NOD2/CARD15 is associated with graft-versus-host disease after hematopoietic stem cell transplantation in children. Biol Blood Marrow Transplant. 2009;15(12):1571-7.

21. van der Velden WJ, Blijlevens NM, Maas FM, Schaap NP, Jansen $\mathrm{JH}$, van der Reijden BA, et al. NOD2 polymorphisms predict severe acute graft-versus-host and treatment-related mortality in T-celldepleted haematopoietic stem cell transplantation. Bone Marrow Transplant. 2009;44(4):243-8. 
22. Holler E, Rogler G, Brenmoehl J, Hahn J, Greinix H, Dickinson $\mathrm{AM}$, et al. The role of genetic variants of NOD2/CARD15, a receptor of the innate immune system, in GvHD and complications following related and unrelated donor haematopoietic stem cell transplantation. Int J Immunogenet. 2008;35(4-5):381-4.

23. Hildebrandt GC, Granell M, Urbano-Ispizua A, Wolff D, Hertenstein B, Greinix HT, et al. Recipient NOD2/CARD15 variants: a novel independent risk factor for the development of bronchiolitis obliterans after allogeneic stem cell transplantation. Biol Blood Marrow Transplant. 2008;14(1):67-74.

24. Granell M, Urbano-Ispizua A, Aróstegui JI, Fernández-Avilés F, Martínez C, Rovira M, et al. Effect of NOD2/CARD15 variants in T-cell depleted allogeneic stem cell transplantation. Haematologica. 2006;91(10):1372-6.

25. Holler E, Rogler G, Herfarth H, Brenmoehl J, Wild PJ, Hahn J, et al. Both donor and recipient NOD2/CARD15 mutations associate with transplant-related mortality and GvHD following allogeneic stem cell transplantation. Blood. 2004;104(3):889-94.

26. Holler E, Rogler G, Brenmoehl J, Hahn J, Herfarth H, Greinix H, et al. Prognostic significance of NOD2/CARD15 variants in HLAidentical sibling hematopoietic stem cell transplantation: effect on long-term outcome is confirmed in 2 independent cohorts and may be modulated by the type of gastrointestinal decontamination. Blood. 2006;107(10):4189-93.

27. Bayston K, Baumgartner JD, Clark P, Cohen J. Anti-endotoxin antibody for prevention of acute GVHD. Bone Marrow Transplant. 1991;8(5):426-7.

28. Moore RH, Lampert IA, Chia Y, Aber VR, Cohen J. Effect of immunization with Escherichia coli $\mathrm{J} 5$ on graft-versus-host disease induced by minor histocompatibility antigens in mice. Transplantation. 1987;44(2):249-53.

29. Gerbitz A, Schultz M, Wilke A, Linde HJ, Schölmerich J, Andreesen R, et al. Probiotic effects on experimental graft-versushost disease: let them eat yogurt. Blood. 2004;103(11):4365-7.

30. Blaser MJ. The microbiome revolution. J Clin Invest. 2014;124(10):4162-5. Review article summarizing $a$ whole series of manuscripts addressing the role of the microbiome and its disturbance in acute infectious diseases, in development of immunity and metabolic diseases, chronic inflammatory disorders as well as oncogenesis.

31.• Jenq RR, Ubeda C, Taur Y, Menezes CC, Khanin R, Dudakov JA, et al. Regulation of intestinal inflammation by microbiota following allogeneic bone marrow transplantation. J Exp Med. 2012;209(5): 903-11. Jenq et al. used murine models of GvHD to describe the impact of the microbiome on GvHD outcome and GvHD-related pathology. With antibiotic pretreatment, they induced shifts in gut microbiome composition and loss of diversity, which translated into increased pathology. Supplemental data on human stool specimens after allogeneic SCT confirm microbiome shifts in relation to human GvHD.

32. Holler E, Butzhammer P, Schmid K, Hundsrucker C, Koestler J, Peter $\mathrm{K}$, et al. Metagenomic analysis of the stool microbiome in patients receiving allogeneic stem cell transplantation: loss of diversity is associated with use of systemic antibiotics and more pronounced in gastrointestinal graft-versus-host disease. Biol Blood Marrow Transplant. 2014;20(5):640-5. In this manuscript, we demonstrated the loss of microbiome diversity after stem cell transplantation using 16 s RNA sequencing, enterococcal PCR and metabolic assessment of indoxyl sulfate. Loss of diversity was associated with use of antibiotics and onset of intestinal GvHD.

33.•- Taur Y, Jenq RR, Perales MA, Littmann ER, Morjaria S, Ling L, et al. The effects of intestinal tract bacterial diversity on mortality following allogeneic hematopoietic stem cell transplantation. Blood. 2014;124(7):1174-82. Taur et al. extend their previous reports to analysis of microbiome diversity early after engraftment following allogeneic stem cell transplantation. They demonstrate a strong impact of loss of diversity on non-relapse related mortality and thus underline the prognostic relevance of microbiome changes.

34. Wehkamp J, Stange EF, Fellermann K. Defensin-immunology in inflammatory bowel disease. Gastroenterol Clin Biol. 2009;33 Suppl 3:S137-44.

35. Bevins CL, Stange EF, Wehkamp J. Decreased Paneth cell defensin expression in ileal Crohn's disease is independent of inflammation, but linked to the NOD2 1007fs genotype. Gut. 2009;58(6):882-3.

36.• Strober W, Asano N, Fuss I, Kitani A, Watanabe T. Cellular and molecular mechanisms underlying NOD2 risk-associated polymorphisms in Crohn's disease. Immunol Rev. 2014;260(1):249-60. Excellent review describing the effect of NOD2 on production of defensins, Paneth cell function, and microbiome composition in health and diseases such as inflammatory bowel disease.

37. Kobayashi KS, Chamaillard M, Ogura Y, Henegariu O, Inohara N, Nuñez G, et al. Nod2-dependent regulation of innate and adaptive immunity in the intestinal tract. Science. 2005;307(5710):731-4.

38. Mortha A, Chudnovskiy A, Hashimoto D, Bogunovic M, Spencer SP, Belkaid Y, et al. Microbiota-dependent crosstalk between macrophages and ILC3 promotes intestinal homeostasis. Science. 2014;343(6178):1249288.

39. Sawa S, Lochner M, Satoh-Takayama N, Dulauroy S, Bérard M, Kleinschek M, et al. ROR $\gamma \mathrm{t}+$ innate lymphoid cells regulate intestinal homeostasis by integrating negative signals from the symbiotic microbiota. Nat Immunol. 2011;12(4):320-6.

40. Hanash AM, Dudakov JA, Hua G, O'Connor MH, Young LF, Singer NV, et al. Interleukin-22 protects intestinal stem cells from immune-mediated tissue damage and regulates sensitivity to graft versus host disease. Immunity. 2012;37(2):339-50. Hanash et al. demonstrate a major role of IL22 in protection of intestinal stem cells. They identify innate lymphoid cells of the recipient as source of IL22 and observed their elimination by GvHD.

41. Cupedo T. Innate protection from graft-versus-host disease. Blood. 2014;124(5):673-5.

42. Furusawa Y, Obata Y, Hase K. Commensal microbiota regulates T cell fate decision in the gut. Semin Immunopathol. 2014 Oct 15. [Epub ahead of print]

43. Singh N, Gurav A, Sivaprakasam S, Brady E, Padia R, Shi H, et al. Activation of Gpr109a, receptor for niacin and the commensal metabolite butyrate, suppresses colonic inflammation and carcinogenesis. Immunity. 2014;40(1):128-39.

44.• Arpaia N, Campbell C, Fan X, Dikiy S, van der Veeken J, de Roos $\mathrm{P}$, et al. Metabolites produced by commensal bacteria promote peripheral regulatory T-cell generation. Nature. 2013;504(7480):4515. Arpaia et al. described the induction of extrathymic Treg cells by butyrate and propionate produced by commensal bacteria in response to starch fermentation. In vivo experiments were supplemented by in vitro Treg expansion assays with butyrate supplementation.

45. Geuking MB, Köller Y, Rupp S, McCoy KD. The interplay between the gut microbiota and the immune system. Gut Microbes. 2014;5(3):411-8.

46. Geuking MB, Cahenzli J, Lawson MA, Ng DC, Slack E, Hapfelmeier S, et al. Intestinal bacterial colonization induces mutualistic regulatory T cell responses. Immunity. 2011;34(5):794806.

47.• Eriguchi Y, Takashima S, Oka H, Shimoji S, Nakamura K, Uryu H, et al. Graft-versus-host disease disrupts intestinal microbial ecology by inhibiting Paneth cell production of $\alpha$-defensins. Blood. 2012;120(1):223-31. Eriguchi et al. reported, for the first time, Paneth cell damage in experimental GvHD. Paneth cell damage is associated with diminished local production of $\alpha$-defensins and subsequent microbiome changes.

48. Ferrara JL, Harris AC, Greenson JK, Braun TM, Holler E, Teshima $\mathrm{T}$, et al. Regenerating islet-derived 3-alpha is a biomarker of 
gastrointestinal graft-versus-host disease. Blood. 2011;118(25): 6702-8.

49. Levine JE, Huber E, Hammer ST, Harris AC, Greenson JK, Braun TM, et al. Low Paneth cell numbers at onset of gastrointestinal graft-versus-host disease identify patients at high risk for nonrelapse mortality. Blood. 2013;122(8):1505-9. Paneth cells have so far not been addressed in histological evaluation of intestinal graft-versushost disease. In these 2 center study, a clear association of Paneth cell loss with histological stage of GvHD was shown, and Paneth cell loss associated with poor outcome of patients suggesting a pathophysiological relevance of intact Paneth cell function.

50. Taur Y, Xavier JB, Lipuma L, Ubeda C, Goldberg J, Gobourne A, et al. Intestinal domination and the risk of bacteremia in patients undergoing allogeneic hematopoietic stem cell transplantation. Clin Infect Dis. 2012;55(7):905-14.

51. Ames NJ, Sulima P, Ngo T, Barb J, Munson PJ, Paster BJ, et al. A characterization of the oral microbiome in allogeneic stem cell transplant patients. PLoS ONE. 2012;7(10):e47628.

52. Rezvani AR, Storb RF. Prevention of graft-vs.-host disease. Expert Opin Pharmacother. 2012;13(12):1737-50.

53. Vossen JM, Guiot HF, Lankester AC, Vossen AC, Bredius RG, Wolterbeek R, et al. Complete suppression of the gut microbiome prevents acute graft-versus-host disease following allogeneic bone marrow transplantation. PLoS ONE. 2014;9(9):e105706.

54. Scott KP, Martin JC, Duncan SH, Flint HJ. Prebiotic stimulation of human colonic butyrate-producing bacteria and bifidobacteria, in vitro. FEMS Microbiol Ecol. 2014;87(1):30-40.

55. Ringdén $\mathrm{O}$, Remberger M, Törlén J, Engström M, Fjaertoft $\mathrm{G}$, Mattsson J, et al. Home care during neutropenia after allogeneic hematopoietic stem cell transplantation in children and adolescents is safe and may be more advantageous than isolation in hospital. Pediatr Transplant. 2014;18(4):398-404.

56. Ringdén O, Remberger M, Holmberg K, Edeskog C, Wikström M, Eriksson B, et al. Many days at home during neutropenia after allogeneic hematopoietic stem cell transplantation correlates with low incidence of acute graft-versus-host disease. Biol Blood Marrow Transplant. 2013;19(2):314-20.

57. Tawara I, Liu C, Tamaki H, Toubai T, Sun Y, Evers R, et al. Influence of donor microbiota on the severity of experimental graft-versus-host-disease. Biol Blood Marrow Transplant. 2013;19(1):164-8.

58. Zhu L, Baker RD, Baker SS. Gut microbiome and non-alcoholic fatty liver diseases. Pediatr Res. 2014 Oct 13. [Epub ahead of print] 